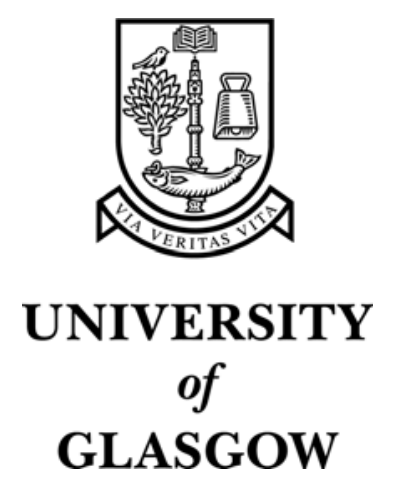

Goodman, J. and Gray, P. and Khammampad, K. and Brewster, S. (2004) Using landmarks to support older people in navigation. Lecture Notes in Computer Science 3160:pp. 38-48.

http://eprints.gla.ac.uk/3283/ 


\title{
Using Landmarks to Support Older People in Navigation
}

\author{
Joy Goodman, Phil Gray, Kartik Khammampad and Stephen Brewster \\ Glasgow Interactive Systems Group, Department of Computing Science \\ University of Glasgow, Glasgow G12 8QQ \\ \{joy, pdg, stephen\}@dcs.gla.ac.uk
}

\begin{abstract}
Although landmarks are an integral aspect of navigation, they have rarely been used within electronic navigation aids. This paper describes the design of a pedestrian navigation aid for a handheld computer, which guides the user along a route using photographs of landmarks, together with audio and text instructions that reference these landmarks. This aid was designed with older users in mind who often find their mobility hampered by declines in sensory, cognitive and motor abilities. It was tested against the standard paper map for the test area with both younger and older people and their performance and subjective workload were measured. The results show that such an aid can significantly outperform a paper-based map and that older people derive substantially more benefit from it than do younger people.
\end{abstract}

\section{Introduction}

The proportion of older people in developed countries is rapidly increasing [13], producing an urgent need to provide greater support for this section of the population. This concern, together with the possibilities and challenges posed by this user group, has prompted recent research into ways in which technology can support and include older people. Much of this research has focused on indoor and stationary applications (see, for example, [14]), but recent advances in handheld computers and positioning technology mean that there is great potential to support older people in mobile situations as well.

Navigation is an important mobile activity, key for maintaining mobility and independence. However, many older people find increasing difficulties with it due to declines in their perceptual, cognitive and motor abilities [8]. This is therefore one area where technology could make a positive difference, through the use of computerised navigation guides and aids.

Current navigation guides usually support navigation by guiding the user along a given route, using turn-by-turn arrow-based directions, or by presenting maps (see, for example, [4]). Some research projects have tried other methods, such as overlaying information on a detailed $1^{\text {st }}$-person view of an area (e.g., [9]), thus allowing reference to specific environmental information, such as landmarks. 
However, landmarks have rarely been used explicitly or in a consistent fashion in navigation guides, although landmarks are an integral aspect of navigation itself [11]. Burnett has shown that their use in vehicle navigation systems can greatly improve their effectiveness [1], but their use within pedestrian navigation guides has not been fully investigated.

This paper describes the design of a pedestrian navigation aid that uses landmarks to help guide the user along a route. This device was designed with older users in mind and used to investigate the feasibility of using landmarks in such a device and the possibilities for how these can be presented. Its performance was compared with that of a paper-based map and age differences in its use were analysed.

\section{The Design of the Navigation Aid}

The design of the navigation aid was informed by some preliminary requirements gathering in the form of focus groups with older people [5]. They answered questions and completed exercises on their travel, navigation and ways of giving directions. Among other results, we found that participants appreciated information about landmarks and liked to be given a visual indication of what these landmarks looked like.

We therefore designed a prototype aid that describes routes using photographs of landmarks. The landmarks were also presented using text and audio directions, so that participants' reactions to the use of landmarks would not be determined by any one particular method of presentation and so that responses to different methods could be gauged.

Ultimately, such an aid would be incorporated into a larger system, allowing users to explore an area and select different start and end points, adapting routes to different users' requirements and coping gracefully when users wander away from the route. It could also be included as part of a larger system that provides, for example, information about the surroundings and places of interest. However, this study focused on the core aspect of such a device (the navigation aid itself) and the methods by which navigation assistance can be best provided.

\subsection{Implementation and Interface Design}

The application was written in C\# and deployed on a Compaq iPAQ. A sample screen is shown on the left in Figure 1. It displays a photograph $(58 \times 43 \mathrm{~mm})$ of a landmark that can be seen from the start of the route. Once the user reaches that location, he or she should press the button labeled "Next Image" to progress to the next instruction and receive a photograph of a new landmark or location to head towards.

As well as the photograph, a brief text instruction is shown and a longer speech instruction can be heard when the "Audio" button is pressed. For example, the speech instruction for the screen in Figure 1 is "From the Western Lecture Theatre, if you look right, you'll see a large chimney. Please go up to it." Audio is presented using the device's built-in speaker. The "View Map" button shows a simplified map of the route as shown on the right in Figure 1. The position of the landmark in the photograph is 
marked with a red dot (dark grey and circled in Figure 1). The "Restart" button returns the user to first screen at the beginning of the route.

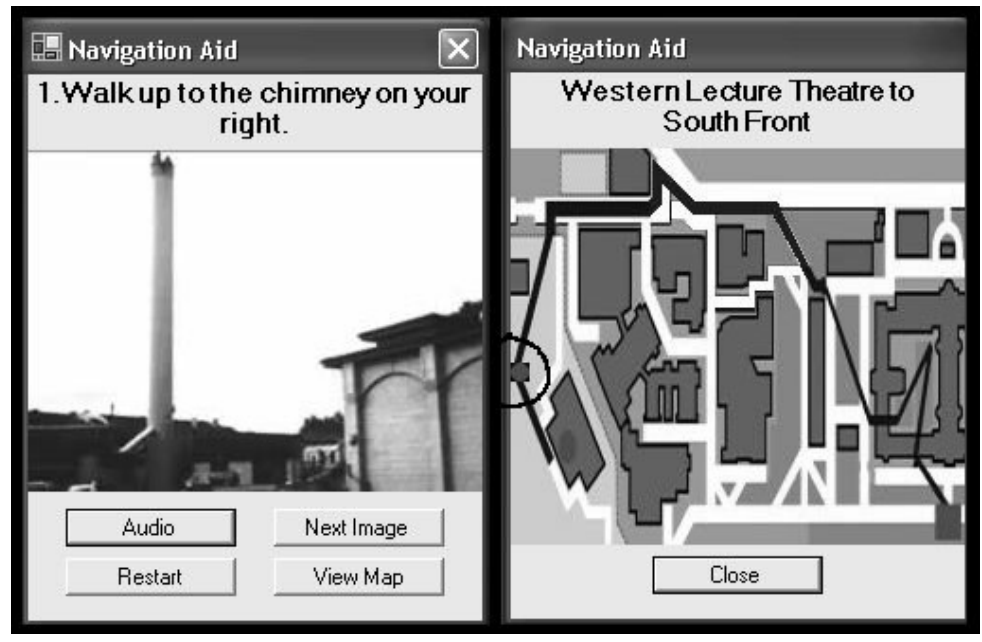

Fig. 1. Example screens from the navigation aid

The interface was designed with guidelines for the design of desktop applications for older adults in mind (e.g., [7]), as little work on mobile design guidelines for older people had been carried out. Although these guidelines had to be adapted to take account of the limited screen size and different interaction techniques, they provided a useful starting point for the design.

For example, drop-down menus and unnecessary features were avoided, text rather than icons was used on buttons and sans-serif fonts were used. A slightly smaller font size (11pt instead of 12pt) than that recommended in [7] had to be used due to the limited screen size available. To compensate for this, a bold typeface was used. In addition, a natural male voice with a standard accent was used for the audio instructions, in line with findings from Lines and Hone [10].

The interface was kept particularly simple for the purposes of testing. In a fully functional system, more attention could be paid to enhancing the aesthetic appeal of the device. However, care must be taken not to compromise the usability of the device, as older people are more easily distracted by un-necessary screen elements and irrelevant information [15].

\section{Evaluation Design}

\subsection{Field Experiments}

The navigation aid was tested using a set of field experiments. Field studies were necessary because the navigation aid is highly dependent upon the surrounding envi- 
ronment and cannot be tested realistically in a laboratory setting. An experimental setup was used to obtain a quantitative comparison of our device against a paper map and of usage by different age groups.

We choose to use an experimental evaluation rather than an ethnographic field study in order to focus on a single aspect of the device (the aiding of navigation) and to obtain quantitative comparisons of performance between different groups of users.

However, field experiments do present the experimenter with several challenges, primarily that of limiting the effect of possibly confounding variables, such as light and noise levels, weather conditions and the time of day. When the levels of such variables cannot be kept consistent, their effects on results can be reduced by varying them across conditions when possible. Removing all variation, however, would produce unrealistic results which may not mean anything for real-world usage. Using real locations and realistic environmental conditions gives real data on how the device is used in practice, and the advantages outweigh the difficulties and make the extra effort worthwhile.

\subsection{Participants}

The navigation aid was tested with 32 able-bodied users; 16 aged between 63 and 77 and 16 between 19 and 34. Each group was balanced with respect to gender. In addition, four "backup" participants were recruited and run through the experiment. This was done in case any data from the main participants was confounded by large changes in the external environment. Only data from one backup participant was actually used because one of the main participants arrived late and so did the experiment in the dark.

In order to avoid over-familiarity with the area, part of the campus of Glasgow University, no participant was either a student or staff member at that university.

All but one of the older participants had never used a hand-held computer before and the remaining participant had only used one a few times. The majority of younger participants (11 out of 16) had also never used a hand-held computer and only one was a regular user. All participants had used a map before with 10 of the younger participants and 11 of the older participants rating themselves as regular map users.

\subsection{Method}

Participants were asked to navigate along two different routes, one of them using the device and the other using the standard paper map for the area. The order of the two routes and the two methods (device or map) were counterbalanced, creating four conditions. Equal numbers from each age group and gender were assigned to each condition.

The experiment was conducted on part of the campus of the University of Glasgow, a common tourist destination within the city of Glasgow. This location was chosen because it has a large number of junctions and decision points in a small area, allowing a sufficiently complicated route to be tested while limiting the length of the routes 
(and therefore of the experiment) in order to avoid tiring participants, particularly the older ones. It also has a low volume of traffic, creating a relatively safe environment, and thus conforming to ethical guidelines.

Routes were chosen within this area with 13-16 waypoints and taking about 10 minutes to walk if walked directly. The sequence of photographs in Figure 2 illustrates a segment of one of the routes used.

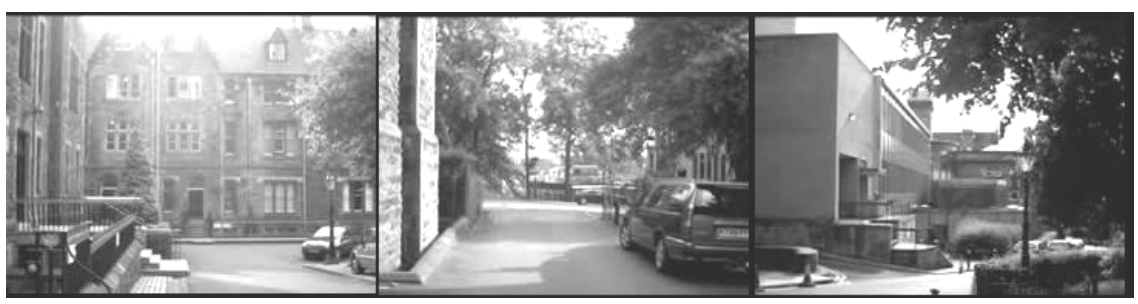

Fig. 2. Images from one of the test routes

\subsection{Map Condition}

The map used was a greyscale version of that available at http://www.gla.ac.uk/general/maps/colourmap.pdf, covering a slightly smaller area and with a shorter list of buildings. A route was indicated on the map using a sequence of numbered, highlighted circles. Part of this map is shown in Figure 3.

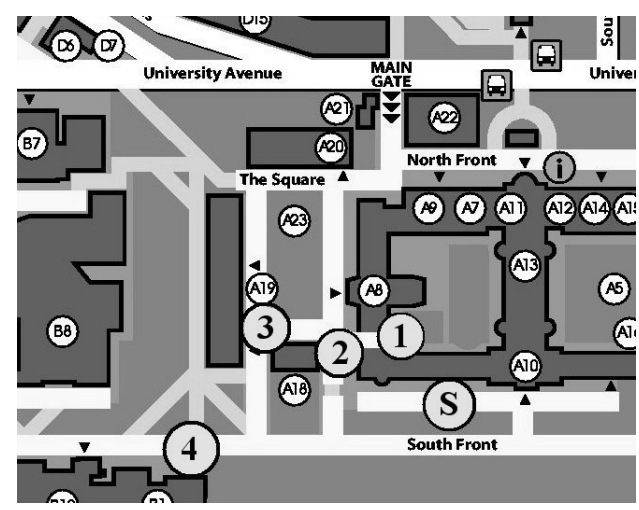

Fig. 3. Part of the map used in the experiment (close to real size)

Participants were asked to navigate along the indicated route, visiting each of the numbered locations in turn. The equivalent route when using the device also passed through each of these locations in the same order. 


\subsection{Procedure}

After an initial briefing, the use of the map or device was explained to participants and they then used this method to find their way along the route. Each participant navigated both routes, using the map on one and the device on the other.

On the routes, the experimenter walked with the participants, a few steps behind them in order not to influence navigation. He or she made written observations on navigation behaviour, as well as providing help when participants got lost. Such help was only provided when it was necessary. Help was given to prevent distress and conform to ethical guidelines, and was noted by the experimenter.

After each route, participants filled in a questionnaire on the device or map. This incorporated the NASA Task Load Analysis (TLX) scale [6], which measures perceived workload, as an indication of how the participants felt about using these methods. We modified the TLX response scales slightly to provide only five possible responses to make them simpler and less daunting for older participants.

The time taken and the number of times that participants got lost on each route were also measured. A participant was defined as lost if the experimenter had to intervene.

\section{Evaluation Results}

\subsection{Timings and Frequency of Getting Lost}

The mean times taken to navigate the routes with the map and the device are shown in Figure 4. A two-way ANOVA on age and method showed a significant main effect of both the navigation method (map or device) and the age group and, perhaps most illuminating, a significant interaction between age and method (all $\mathrm{p}<0.001)$.

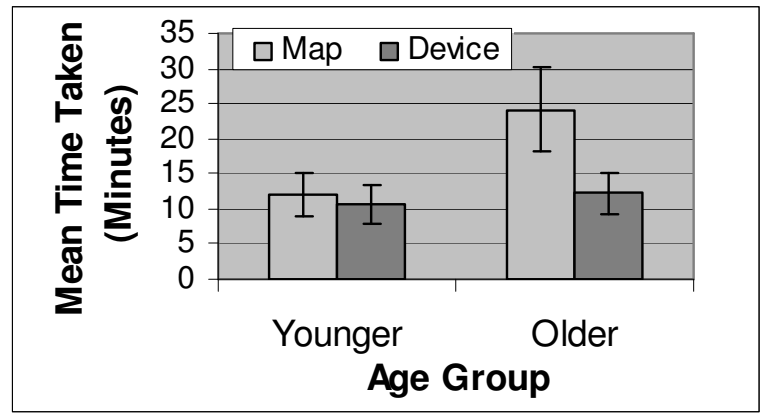

Fig. 4. Mean time taken to navigate test routes (error bars show standard deviation)

The navigation method affects the time taken; participants took significantly less time when they used the navigation aid than when they used the map. Age group also affects the time, with younger participants being significantly faster. 
However, both of these effects do not really describe the results shown in Figure 4. The interaction between the different factors gives a much clearer insight into the results. An analysis of the interaction showed that the time differences between the age groups are only significant with the map, not with the device, and that only the older sample displays a significant difference between the map and the device $(\mathrm{p}<0.001$, $\mathrm{t}$ tests).

Participants also got lost significantly less often with the device $\left(\mathrm{p}<0.001, \mathrm{t}_{\text {-test }}{ }^{1}\right)$, where "lost" is as defined above. In fact, no participants got lost when using the device, compared to a mean of 1.9 times per route for older users and 0.4 times for younger users when using the map.

\subsection{TLX Scores}

Raw TLX (RTLX) scores were calculated as a measure of overall workload [2]. These were significantly lower for the device than for the map $(\mathrm{p}<0.001$, Mann-Whitney) and there was no significant effect of age group ( $p>0.05)$. The TLX scores can be further investigated by analysing their individual components as shown in Figure 5. Because we simplified the TLX scales, these scores were calculated out of 5 instead of 20 .

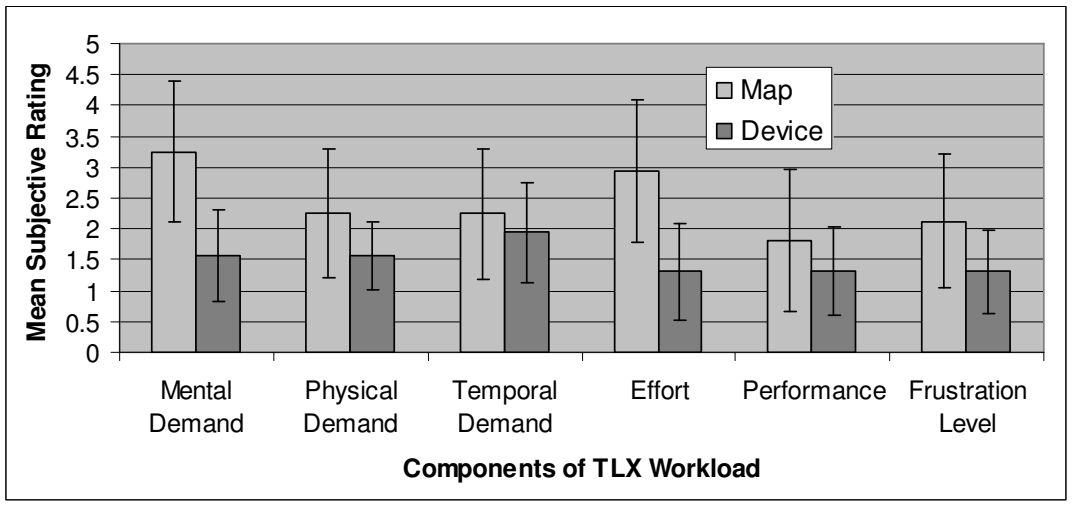

Fig. 5. Mean TLX Scores for the map and navigation aid. Higher values indicate higher workload and lower performance. Error bars show standard deviation

Using Mann-Whitney tests, significant differences between the map and the device were found for mental demand, effort and frustration $(\mathrm{p}<0.001)$, as well as for performance $(\mathrm{p}<0.005)$ and physical demand $(\mathrm{p}<0.05)$. There was no significant difference in temporal demand $(\mathrm{p}>0.05)$.

${ }^{1}$ Although the frequency of getting lost is non-parametric, a t-test was used because tests such as Mann-Whitney could not be applied since all of the results for one of the conditions (use of the device) are identical (all are 0 ). In this case, the results from the t-test are significant enough and a t-test robust enough to conclude that a significant difference does exist. 


\subsection{Preferences and Comments}

After trying both methods, participants were asked to indicate on a 5-point scale which method they found most useful. The results are shown in Figure 6 below. Only one person (an older user) indicated a preference for the map, explaining that she was "accustomed to using maps and feels comfortable with them".

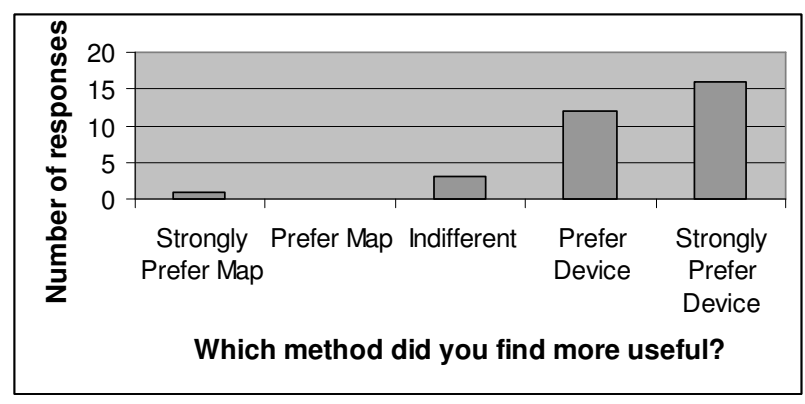

Fig. 6. Perceived relative usefulness of the map and the navigation aid

Reasons given for preferring the device were varied, with some people giving multiple reasons. Most commonly mentioned was the provision of images of locations, which some said helped them to confirm where they were or to determine more easily where they should go. Some participants liked having step-by-step directions and one user said this was like "walking with a guide who knew each and every corner".

Other reasons focused on the shortcomings of maps, with both difficulties with maps in general and specific shortcomings of the map provided being mentioned. One participant explained that "using maps in general is quite difficult", while another described how he "would have to turn and try and work out which way using the map". A few people mentioned shortcomings of the particular map used; for example, one person complained that there was no indication that a grey area represented a car park.

Despite the preferences for the device, some of the participants did have some reservations about it. Some felt that a map would be better for longer routes; others that the device gives less freedom and control over the route and a poorer idea of the route as a whole. Nevertheless, the majority felt the device to be more useful.

\section{Discussion}

This work demonstrates that landmarks can be used effectively to support navigation through a handheld device. Such a device can improve the time taken to navigate a route and reduce the number of times when people get lost, compared to a paper map. It can also reduce workload and users agree that the device is more useful than the map. 
Furthermore, such a device has a greater potential benefit for older users than for younger. Although both older and younger users found the device useful (they got lost less often with the device, found that it produced a smaller workload and felt that it was more useful than the map), only older participants completed routes faster when using the device.

It may have been expected that older participants would have difficulty using a handheld device, particularly because all but one had never used one before. However, participants had little difficulty using the navigation aid and gave it low ratings on all aspects of TLX workload. We found that if the interface on a handheld computer is carefully designed with older people in mind, then it can be used without difficulty by this age group. This agrees with results from other studies, such as [12]. In [12], McGee et al found that, when the interface for a handheld application for cancer patients was redesigned based on the results of pilot studies, it was used without difficulty by the user group, many of whom were in the older age category.

We also expected older participants to be slower than younger ones, due to reduced walking speed, but this effect was only observed with the map. A landmark-based device has the potential to improve older people's performance to a level comparable with a younger age group.

This does not mean that such a device would not be useful for any younger person. Although the younger group as a whole did not experience significant improvement with the device, individuals did. We cite the example of one of the backup participants (one of the younger group) who took over three times as long and got lost 7 times with the map, as opposed to once at the start when using the device.

The use of the device can be investigated further by examining the components of the TLX scores. As well as increasing efficiency, the device has the advantage of decreasing the mental and physical demand, the effort expended and the frustration experienced. Users were also aware of an increase in their performance level.

There are a variety of possible reasons for this increase in performance and decrease in workload. While this study cannot give any definitive answers, some indications can be gathered from participants' comments. When asked to explain why they preferred the device, several participants (both older and younger) explained that it gave a visual identification or confirmation of locations on the route. Several also liked being given a set of directions and being told which direction to turn in rather than having to figure it out from a map.

All of this does not mean that there are no difficulties with a landmark-based navigation aid. The step-by-step nature of such an aid reduces the user's freedom and control and provides a poorer overall idea of the route. There is also a degree of natural resistance to new methods. Research is needed into ways to overcome these challenges, e.g., by providing support for the user to change the route.

\section{Future Work}

Although we have demonstrated that landmarks can be used effectively in our test area, this area is only representative of a subset of possible locations. Landmark-based 
navigation aids also need to be tested in environments such as city centres and shopping areas, which have different kinds of street layouts and in which people may be doing different kinds of activities. Similarly, the map used in this study was the standard map provided by the university to its visitors. While similar in type to a street map, there were some significant differences, as it used a simplified and stylised design, rather than being topologically correct. It is important to see how navigation aids compare with standard street maps.

It is also unclear how much of the success of the navigation aid can be ascribed to different factors, such as the electronic nature of the aid, the step-by-step directions, the images of landmarks and the verbal and written instructions. In particular, there are many ways of presenting landmarks to users. The aid used in this study did this through photographs, text and speech in an electronic medium, but other methods are possible. It is important to examine the different methods and modalities to determine which are most effective in aiding navigation.

As a first step towards this, we have modified the navigation aid from this study to provide a choice of different methods of presenting the information: as well as a combination of photographs, text and speech, there is a text only, a speech only and a text and speech interface. We are currently conducting a set of field experiments, comparing these last three versions of the interface, in order to analyse the contribution of each modality.

We also hope to investigate ways in which a navigation aid can help users to gain a better overall idea of a route and area, perhaps enabling them to explore a location rather than follow specific routes. In the course of this, we plan to consider issues of users' freedom and control over their navigation and the routes that they take.

\section{Conclusions}

Landmarks are a key part of navigation and this study has shown that they can be used effectively within electronic pedestrian navigation aids. A device that bases its navigation guidance around landmarks can significantly outperform a paper-based map, as well as reducing subjective workload and eliciting a positive response from users.

In addition, we have found that older people derive substantially more benefit from such a device than do younger users, with a large reduction in the time taken to navigate routes. The use of handheld technology does not prevent them from using the navigation aid successfully. Such aids could, therefore, provide key support to older people in maintaining their mobility and independence.

\section{Acknowledgements}

This work was funded by SHEFC through the UTOPIA project (grant number: HR01002), investigating the design of usable technology for older people [3]. We would like to thank Morag Stark for helping to conduct some of the experiments and also all those who took part in the experiments. 


\section{References}

1. Burnett, G.E.: "Turn right at the traffic lights" The requirement for landmarks in vehicle navigation systems. The Journal of Navigation 53(3) (2000) 499-510

2. Byers, J.C., Bittner, Jr, A.C. and Hill, S.G.: Traditional and Raw Task Load Index (TLX) Correlations: Are Paired Comparisons Necessary? In: Mital, A. (ed.): Advances in Industrial Ergonomics and Safety I, Taylor and Francis (1989) 481-485

3. Eisma, R., Dickinson, A., Goodman, J., Syme, A., Tiwari, L., Newell, A.F.: Early User Involvement in the Development of Information Technology-Related Products for Older People. To appear in Design Principles to Support Older Adults, Special Issue of Universal Access in the Information Society 3(2), to appear 2004

4. Garmin. http://www.garmin.com

5. Goodman, J., Dickinson, A., Syme, A.: Gathering Requirements for Mobile Devices using Focus Groups with Older People. To appear in Designing a More Inclusive World, Proceedings of the 2nd Cambridge Workshop on Universal Access and Assistive Technology (CWUAAT), Springer (2004)

6. Hart, S.G., Staveland, L.E.: Development of NASA-TLX (Task Load Index): Results of Empirical and Theoretical Research. In: Hancock, P., Meshkati, N. (eds.): Human Mental Workload. North Holland B.V., Amsterdam (1988) 139-183

7. Hawthorn, D.: Possible implications of aging for interface designers. Interacting with Computers 12(5) (2000) 507-528

8. Kirasic, K.C.: Ageing and spatial behaviour in the elderly adult. In: Kitchin, R., Freundschuh, S. (eds.): Cognitive Mapping: Past, present and future. Routledge, Routledge Frontiers of Cognitive Science 4 (2000) Chapter 10

9. Laakso, K., Gjesdal, O., Sulebak, J.R.: Tourist information and navigation support by using 3D maps displayed on mobile devices. Proceedings of HCI in Mobile Guides, Workshop at Mobile HCI 2003 (2003) 34-39

10. Lines, L., Hone, K.S.: Older Adults' Evaluations of Speech Output, Proceedings of Assets 2002, ACM Press (2002) 170-177

11. Lynch, K.: The Image of the City. MIT Press, Cambridge, Mass. (1960)

12. McGee, M., Gray, P., Muir, L, Hargan, I: Designing a Chemotherapy Symptom Management System for PDAs: Emerging Design Guidelines from Early Patient Pilot Studies. Technical report TR-2003-146, Department of Computing Science, University of Glasgow (2003)

13. U.S. Census Bureau, International Data Base (IDB). http://www.census.gov/ipc/www/ idbnew.html, updated $17^{\text {th }}$ July 2003

14. Zajicek, M., Brewster, S. (eds.): Design Principles to Support Older Adults, Special Issue of Universal Access in the Information Society 3(2), to appear April 2004

15. Zec, R.F.: The Neuropsychology of Aging. Experimental Gerontology 30(3-4) (1995) 431442 